\title{
Antioxidants Distribution in Pulp and Seeds of Black and Red Goji Berries as Affected by Boiling Processing
}

\author{
Guang Xin, ${ }^{1}$ Fengmei $\mathrm{Zhu},{ }^{2}$ Bin $\mathrm{Du}^{2}$ and Baojun $\mathrm{Xu}^{3}$ \\ ${ }^{1}$ College of Food Science, Shenyang Agricultural University, Shenyang 110161, China \\ ${ }^{2}$ Hebei Normal University of Science and Technology, Qinhuangdao, Hebei 066600, China \\ ${ }^{3}$ Food Science and Technology Program, Beijing Normal University-Hong Kong Baptist University United International College, \\ Zhuhai, Guangdong 519085, China
}

Correspondence should be addressed to Baojun Xu; baojunxu@uic.edu.hk

Received 1 July 2017; Revised 15 September 2017; Accepted 1 October 2017; Published 24 October 2017

Academic Editor: Isabela M. Toaldo

Copyright (C) 2017 Guang Xin et al. This is an open access article distributed under the Creative Commons Attribution License, which permits unrestricted use, distribution, and reproduction in any medium, provided the original work is properly cited.

Pulp and seeds from four varieties of black goji and two varieties of red goji with different geographical origins were examined for their total phenolic content (TPC), total flavonoid content (TFC), condensed tannin content (CTT), monomeric anthocyanin content (MAC), 2,2-diphenyl-1-picrylhydrazyl (DPPH) scavenging capacity, and ferric reducing antioxidant power (FRAP) using colorimetric methods. Contribution rates of pulp and seed to phytochemical contents and overall antioxidant capacities of whole fruits were calculated for each parameter. It was observed that most of the phytochemicals and antioxidant activities were predominantly contributed by the pulp in all six varieties. Boiling led to significant $(p<0.05)$ losses in the phytochemical content and antioxidant capacity. The average MAC value in black goji was significantly $(p<0.05)$ higher than that observed for red goji.

\section{Introduction}

As the fruits of Solanaceae family shrub plant, goji (also called as wolfberry) has been used as traditional medicinal foods in China and other Asian countries for centuries [1]. Red goji (Lycium barbarum L.) is a perennial, deciduous shrub growing northwest China and the Mediterranean region [2]. Black goji (heiguogouqi in Chinese) is a black color small berry fruit from (Lycium ruthenicum Murr.) natively growing in northwest part of China. Goji is a good source of fiber, protein, carotenoids [3], and polysaccharides [4]. Recent research indicates that goji had a lot of biological activities, including antidiabetes [5], antiproliferative activity [4], preserving retinal function [6], and antioxidant activity $[7,8]$. A lot of research has focused on carotenoids [3] and polysaccharides $[4,6]$. However, phytochemicals $[9,10]$, such as phenolic compounds [11], including phenolic acids and flavonoids in goji and their antioxidant activity have been less evaluated.

Furthermore, the information on the effect of goji color and the effect of boiling on phytochemical distribution of goji is very few. The aim of this work was to compare the phytochemicals and antioxidant activities between red and black goji, discuss the distribution of phytochemicals in fruits, and investigate the contribution of goji pulp and seeds to the antioxidants of whole goji.

\section{Material and Methods}

2.1. Experimental Materials and Chemicals. Four varieties of black goji and two varieties of red goji were used to carry out this work (Table 1, Figure 1). The varieties varied in their places of origin and were bought from six different sources.

Folin-Ciocalteu reagent was purchased from Beijing Aoboxing Biotechnology Co., Ltd. (Beijing, China). Gallic acid, (+)-catechin, vanillin, 1,1-diphenyl-2-picrylhydrazyl (DPPH), and 2,4,6-tri(2-pyridyl)-striazine (TPTZ) were purchased from the National Standard Samples Center (Beijing, China). All other reagents were of analytical grade.

2.2. Preparation of Goji Sample. The goji samples preparation was conducted using a modified colorimetric method [12]. 
TABLE 1: Source of origin, color, and sample ID of goji berry samples.

\begin{tabular}{lcc}
\hline Variety number & Variety type and name & Place of origin \\
\hline 1 & Xinjiang black goji & Kashi, Xinjiang, China \\
2 & Ningxia black goji & Yinchuan, Ningxia, China \\
3 & Tibet black goji & Linzhi, Xizhang, China \\
4 & Qinghai black goji & Geermu, Qinghai, China \\
5 & Ningxia red goji (big fruits) & Yinchuan, Ningxia, China \\
6 & Ningxia red goji (small fruits) & Yinchuan, Ningxia, China \\
\hline
\end{tabular}

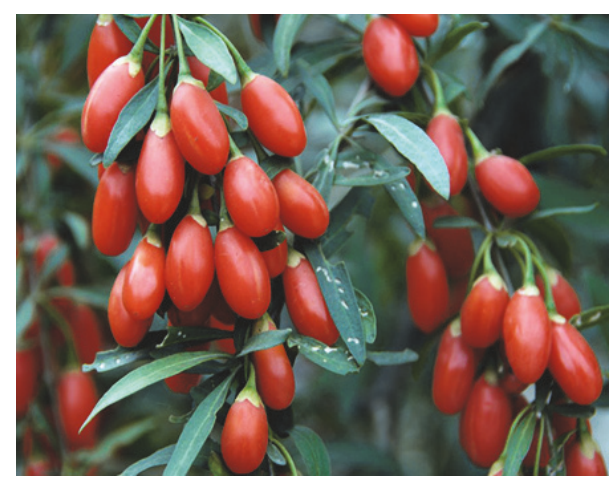

(a)

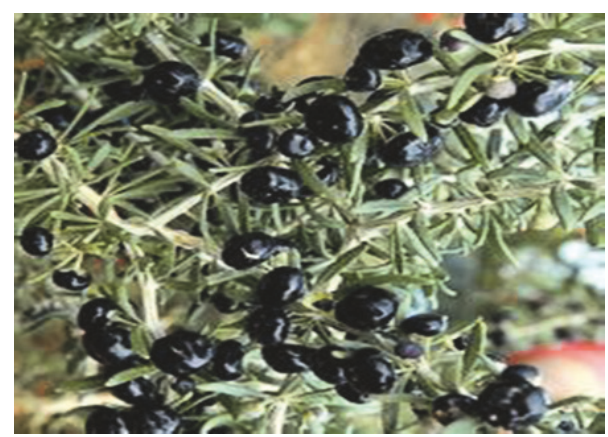

(c)

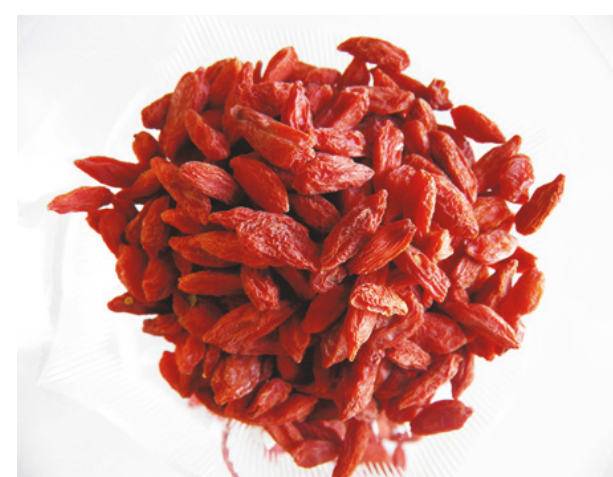

(b)

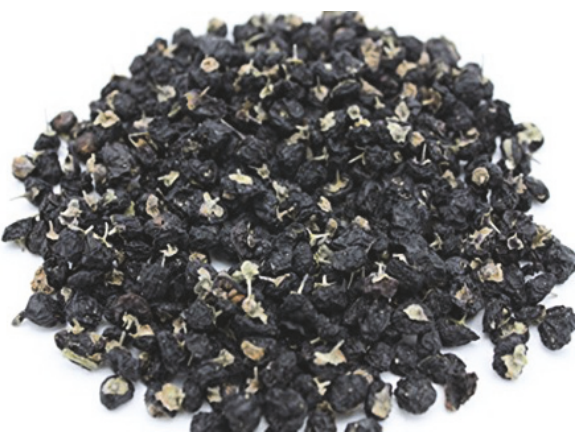

(d)

FIGURE 1: The black and red goji berries. (a) Fresh red goji (Lycium barbarum L.); (b) dry red goji; (c) fresh black goji (Lycium ruthenicum Murr.); (d) dry black goji.

2.3. Extraction of Phytochemicals from Black and Red Goji. Each powdered sample $(1.0000 \mathrm{~g})$ was taken in a capped centrifuge tube and extracted twice using $30 \mathrm{~mL}$ of $60 \%(\mathrm{~V} / \mathrm{V})$ ethanol. The mixture was extracted for $30 \mathrm{~min}$ using a Scientz-IID ultrasonic cell disrupter (Ningbo Scientz Biotechnology Company, Ltd., Ningbo, Zhejiang, China). The extract was centrifuged at $5000 \mathrm{rpm}$ for $10 \mathrm{~min}$ and collected in a new centrifugal tube. The extraction was carried out in triplicates and extracts were kept at $4^{\circ} \mathrm{C}$ in the dark for next study.

2.4. Determination of Total Phenolic Content (TPC). The TPC of samples was determined using the Folin-Ciocalteu reagent-based colorimetric assay as described by Dong et al. [13]. Phenolic content was calculated as mean (gallic acid equivalents (GAE) and reported as $\mathrm{mg} \mathrm{g}^{-1}$ sample) \pm standard deviation (SD) for three replications.
2.5. Determination of Total Flavonoid Content (TFC). The TFC of samples was determined using a modified colorimetric method $[10,12]$. The flavonoid content was determined by a catechin standard curve and expressed as the mean (milligrams of $(+)$-catechin equivalents (CAE) per g sample) \pm standard deviation (SD) for three replications.

2.6. Determination of Monomeric Anthocyanin Content (MAC). MAC was measured using a $\mathrm{pH}$ differential method as described by Lee et al. [14]. The MAC results were reported as cyanidin-3-glucoside equivalents ( $\mathrm{mg} \mathrm{CyE} / \mathrm{g}$ sample) using a molecular weight of $449.2 \mathrm{~g} \mathrm{~mol}^{-1}$ and a molar extinction coefficient of $26,900 \mathrm{~L} \mathrm{~cm}^{-1} \mathrm{~mol}^{-1}$.

2.7. Determination of Condensed Tannin Content (CTC). CTC was determined using a modified colorimetric method as described by Xu and Chang [15]. The results were expressed 
TABLE 2: The physical properties of different goji varieties.

\begin{tabular}{lcrr}
\hline Variety number & Weight of 100 raw goji (g) & \multicolumn{2}{c}{ Distribution of weight (\%) } \\
\hline 1 & $4.12 \pm 1.78$ & $54.85 \pm 4.31$ & $45.15 \pm 3.88$ \\
2 & $2.50 \pm 0.47$ & $55.87 \pm 3.77$ & $44.13 \pm 5.30$ \\
3 & $3.14 \pm 0.77$ & $40.29 \pm 7.63$ & $59.71 \pm 4.36$ \\
4 & $3.67 \pm 0.47$ & $43.35 \pm 6.59$ & $56.65 \pm 6.05$ \\
5 & $23.37 \pm 4.57$ & $83.88 \pm 5.94$ & $16.12 \pm 2.09$ \\
6 & $10.16 \pm 2.39$ & $74.88 \pm 7.30$ & $25.12 \pm 3.33$ \\
\hline
\end{tabular}

as $(+)$-catechin equivalents (mg CAE/g sample) by using a calibration curve of $(+)$-catechin with linearity range from 50 to $1000 \mu \mathrm{g} / \mathrm{mL}$.

2.8. Determination of Ferric Reducing Antioxidant Power (FRAP). FRAP was measured using a colorimetric method as described by $\mathrm{Zhu}$ et al. [16]. The results were expressed as micromoles of $\mathrm{Fe}^{2+}$ equivalents per gram of sample ( $\mu \mathrm{mol} \mathrm{Fe} \mathrm{F}^{2+} / \mathrm{g}$ sample) using a calibration curve of $\mathrm{Fe}^{2+}$ with linearity range of $10-1000 \mu \mathrm{mol} / \mathrm{L}$.

2.9. Determination of Free Radical Scavenging Activity $(D P P H)$. DPPH radical scavenging capacities of samples were evaluated according the method of Tanvir et al. [17] with slight modifications. The DPPH was expressed as the mean \pm standard deviation (SD) for three replications.

2.10. The Contribution Rate of Pulp and Seeds in Goji Fruits. The goji fruits consist of pulp and seeds, so contribution of these two components to the fruits was calculated in contribution rates way. The contribution rates were calculated as the following equation:

$$
\begin{aligned}
& \mathrm{CR}_{A}(\%)=C_{\text {target substance } A} \\
& \times \frac{W_{A}(\%)}{\left[C_{\text {target substance } A} * W_{A}(\%)+C_{\text {target substance } B} \times W_{B}(\%)\right]},
\end{aligned}
$$

where $\mathrm{CR}_{A}(\%)$ is the contribution rate of $A$ (\%); $C_{\text {target substance } A}$ is the content of target substance in $A$; $W_{A}(\%)$ is the percentage weight of $A$ in the sample; $C_{\text {target substance } B}$ is the content of target substance in $B ; W_{B}$ (\%) is the percentage weight of $B$ in the sample.

2.11. Statistical Analysis. All results were expressed as mean $\pm \mathrm{SD}$ of three replicates. Data in triplicate were analyzed by one-way analysis of variance and Duncan's multiple range test using SPSS 11.5 software package for Windows (IBM Corporation, Armonk, NY, USA). Data with $p<0.05$ were considered as significantly different [18].

\section{Results and Discussion}

3.1. Physical Properties of Black and Red Goji. The physical properties of all the six varieties considered in the study have been summarized in Table 2 . The average weight of 100 grains varied from 2.5 to $4.1 \mathrm{~g}$ for black goji. Pulp and seeds contributed equally (about 50\%) to the total fruits weight. For red goji, the average weight of 100 grains varied from 10.1 to $23.3 \mathrm{~g}$. Of this, pulp occupied more than $75 \%$ of the total weight for red goji with the highest value observed for variety number 3 at $83.8 \%$.

3.2. Contents of Phytochemicals in Black and Red Goji. Spectrophotometric method is one good method to assess the major phenolic classes in plants [19]. TPCs content of the different parts of black and red goji have been indicated. The results showed that TPC was significantly $(p<0.05)$ higher in pulp but comparable in seeds and fruits for all the varieties (Table 3). For each cultivar, the TPC content tendency of different parts was the same as follows: pulp > seeds $>$ whole fruits. TPCs for the pulp for different varieties ranged from 3.44 to $6.54 \mathrm{mg} \mathrm{GAE} / \mathrm{g}$, while for seeds, the values obtained were 1.19-3.37 mg GAE/g (Table 4). The TPC found in our study was similar to data reported in the literature [20]. They presented that the TPC in fresh and dried goji fruits are 174.27 and 342.59 gallic acid equivalents/100 g, respectively, using ethanol $80 \%$ as extract solvent. Moreover, the other group measured a mean TPC of $8.65 \mathrm{~g} \mathrm{GAE} / 100 \mathrm{~g}$ seeds in Jabuticaba (Myrciaria cauliflora) seeds [21].

For the same part among different varieties, the TPCs were found to be significantly $(p<0.05)$ different (Table 4$)$. As far as the contribution rates are concerned, pulp contributed to majority of TPC (57-93\%) presented in goji. Only 7\%-43\% of the TPC could be allocated to the seeds (Table 5).

TFC in pulp was significantly $(p<0.05)$ different for all the varieties considered in the study (Table 3 ). Moreover, pulp had the highest TFC values, followed by seeds and whole fruits for all six varieties. For pulp, the values ranged from 2.2 to $11.6 \mathrm{mg} \mathrm{CAE} / \mathrm{g}$, while, for seeds, the values were found to be between 0.26 and $2.37 \mathrm{mg} \mathrm{CAE} / \mathrm{g}$ and, for fruits, the values were $0.12-1.24 \mathrm{mg} \mathrm{CAE} / \mathrm{g}$. And, for red and black variety, four black goji samples had a very high concentration of TFCs than red samples. The results showed that the contribution rate of the pulp to the TFC was high. There is similar tendency in the previous publication [8]. The contribution rate of the seeds $(9-22 \%)$ to the TFC of the whole goji was significantly $(p<0.05)$ lower as compared to that of the pulp $(78-91 \%)$ (Table 5).

The results showed that for five different varieties except variety 1 , pulp had the highest concentration of MAC. The values obtained for variety 4 (black goji) were significantly 
大o| ڤ $\pi+1+1+1+1+1+1+1+1+1+1+1+1+1+1+1+1+1+1+1+1+1+1+1+1$

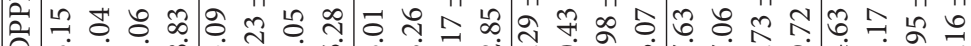
A

कo

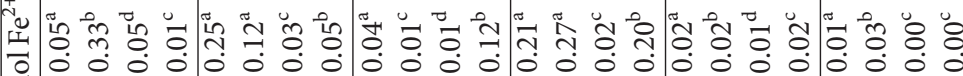
$1+1+1+1+1+1+1+1+1+1+1+1+1+1+1+1+1+1+1+1+1+1+$

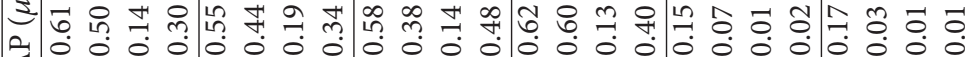

空

空

, 50

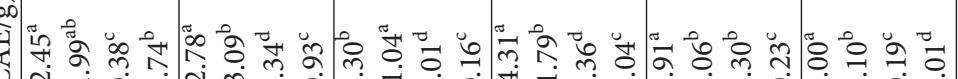

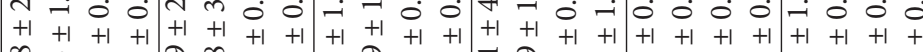
U)

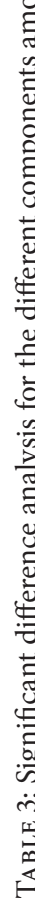

कon

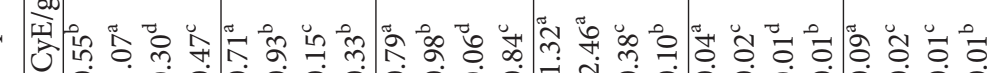

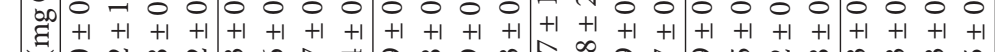

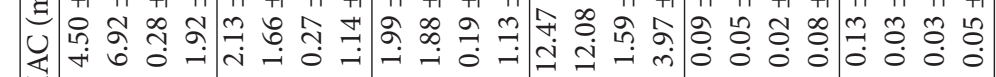

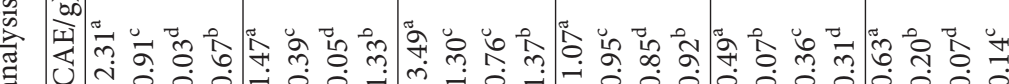

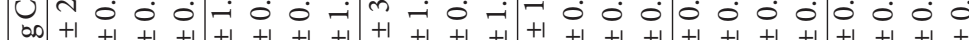

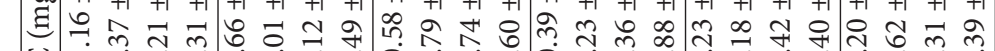

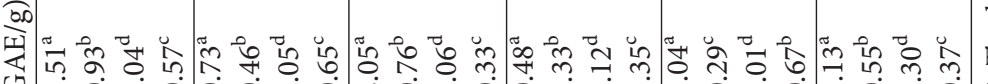

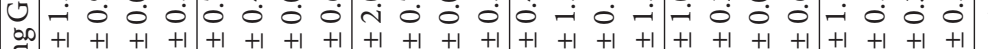
可诲

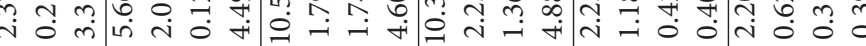

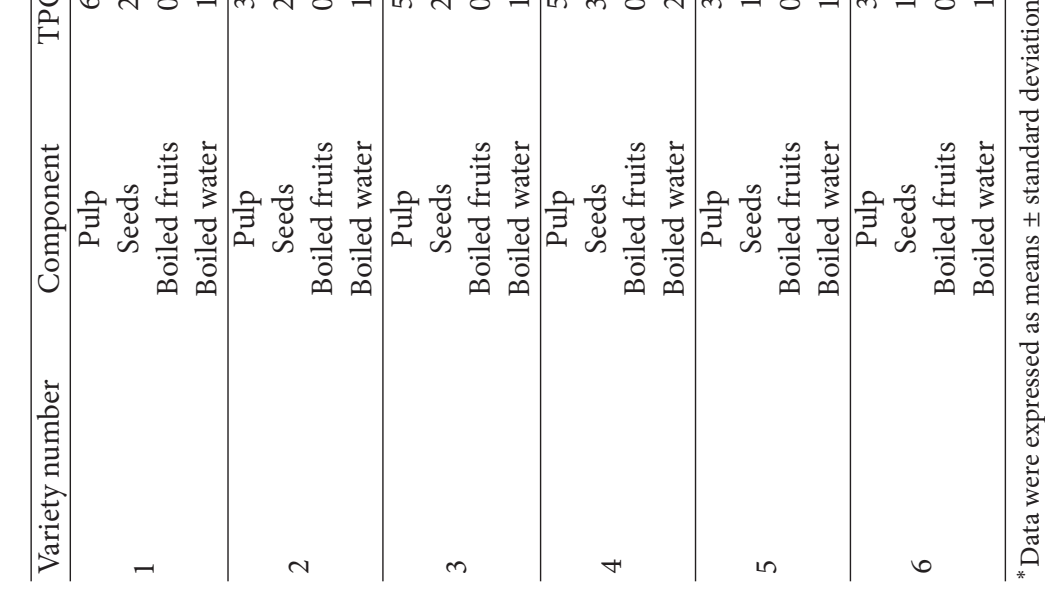




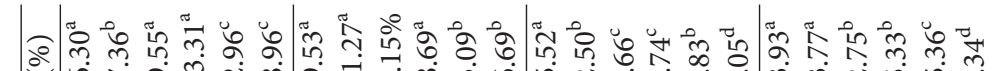
○| $1+1+1+1+1+1+1+1+1+1+1+1+1+1+1+1+1+1+1+1+1+1+1+1$ 节 要

कon

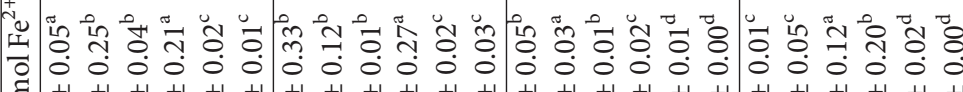
F $+1+1+1+1+1+1+1+1+1+1+1+1+1+1+1+1+1+1+1+1+1+1+1+$

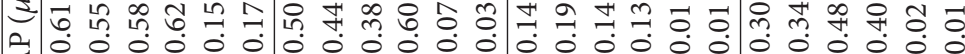

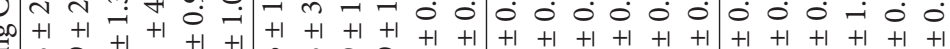

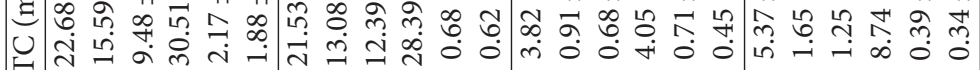

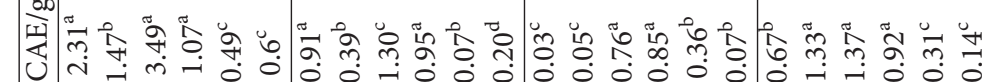
$300+1+1+1+1+1+1+1+1+1+1+1+1+1+1+1+1+1+1+1+1+1+1+1$ U్ర

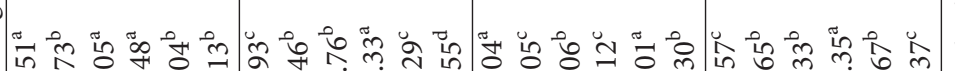

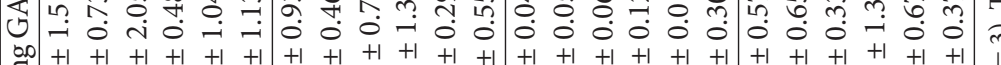

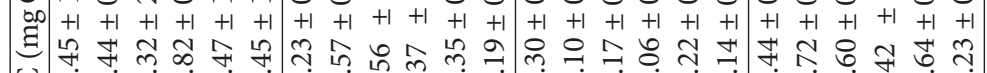

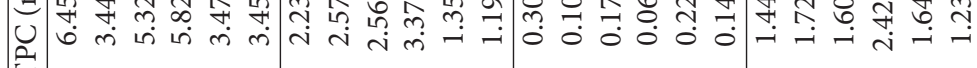


TABLE 5: Contribution rates of pulp and seeds to the overall phytochemical content and antioxidant activity of goji.

\begin{tabular}{|c|c|c|c|}
\hline \multirow{2}{*}{ Antioxidants } & \multirow{2}{*}{ Variety number } & \multicolumn{2}{|c|}{ Contribution rate (\%) } \\
\hline & & Pulp & Seeds \\
\hline \multirow{6}{*}{ ТPC } & 1 & 78 & 22 \\
\hline & 2 & 63 & 37 \\
\hline & 3 & 58 & 42 \\
\hline & 4 & 57 & 43 \\
\hline & 5 & 93 & 7 \\
\hline & 6 & 90 & 10 \\
\hline \multirow{6}{*}{ TFC } & 1 & 85 & 15 \\
\hline & 2 & 78 & 22 \\
\hline & 3 & 80 & 20 \\
\hline & 4 & 78 & 22 \\
\hline & 5 & 91 & 9 \\
\hline & 6 & 91 & 9 \\
\hline \multirow{6}{*}{ MAC } & 1 & 44 & 56 \\
\hline & 2 & 62 & 38 \\
\hline & 3 & 42 & 58 \\
\hline & 4 & 44 & 56 \\
\hline & 5 & 90 & 10 \\
\hline & 6 & 93 & 7 \\
\hline \multirow{6}{*}{ CTC } & 1 & 56 & 44 \\
\hline & 2 & 60 & 40 \\
\hline & 3 & 34 & 66 \\
\hline & 4 & 45 & 55 \\
\hline & 5 & 90 & 10 \\
\hline & 6 & 93 & 07 \\
\hline \multirow{6}{*}{ FRAP } & 1 & 60 & 40 \\
\hline & 2 & 61 & 39 \\
\hline & 3 & 51 & 49 \\
\hline & 4 & 44 & 56 \\
\hline & 5 & 92 & 8 \\
\hline & 6 & 94 & 6 \\
\hline \multirow{6}{*}{ DPPH } & 1 & 72 & 28 \\
\hline & 2 & 63 & 37 \\
\hline & 3 & 54 & 46 \\
\hline & 4 & 62 & 38 \\
\hline & 5 & 94 & 6 \\
\hline & 6 & 92 & 8 \\
\hline
\end{tabular}

$(p<0.05)$ higher than those for other samples. The values for pulp ranged from 0.09 to $12.4 \mathrm{mg} \mathrm{CyE} / \mathrm{g}$. They were minimum for variety 5 (red goji). The seeds were between 0.03 and $12.08 \mathrm{mg} \mathrm{CyE} / \mathrm{g}$ and were minimum for variety 6 (red goji), while for fruits, the values were $0.02-1.57 \mathrm{mg} \mathrm{CyE} / \mathrm{g}$. Anthocyanins are colored pigments, purple or black in color [12]. Hence, the MAC for black goji is considerably higher than that observed for red goji. For black goji (variety $1,3,4$ ), the contribution rate of the seeds was significantly $(p<0.05)$ higher than the pulp (Table 5).

CTC in the pulp, seeds, and fruits for all six goji varieties has been listed in Table 3. The results showed that pulp had the highest contribution for all six varieties. The values were the highest for pulp and the lowest for fruits in between them. Interestingly, CTC content in different parts had the following tendency: pulp > seeds > whole fruits. Interestingly, for each variety, $34-94 \%$ of the CTCs were contributed by the pulp.

3.3. Antioxidant Activities (FRAP and DPPH) of Black and Red Goji. Pulp had the highest FRAP values in six different varieties. FRAP values were the lowest for fruits for all the black and red goji (Table 3). For each variety, the trend of FRAP value for the different components was observed to be as follows: pulp > seeds > whole fruits. Across different varieties, FRPA values for the same part were found to be significantly $(p<0.05)$ different but comparable (Table 4$)$. The values for pulp lied between 0.15 and $0.62 \mu \mathrm{mol} \mathrm{Fe}^{2+} / \mathrm{g}$ and the same for seeds which were found to be lying between $\mu \mathrm{mol} \mathrm{Fe} e^{2+} / \mathrm{g}$. As far as the total contribution of each component to the overall antioxidant activity of the whole goji is concerned, the results indicated that the contribution rates of both pulp and seeds were comparable (Table 5). While the contribution rate of pulp was the lowest for variety 6 (44\%), it was the highest for variety 4 (94\%).

DPPH free radical scavenging activity was the highest for pulp compared to seeds $(p<0.05)$ in all six varieties (Table 3 ). The tendency of DPPH results from different parts was pulp $>$ seeds $>$ whole fruits which is the same trend as obtained using the FRAP assay also. For pulp, DPPH free radical scavenging activity ranged from 44.6 to $85.1 \%$, while for seeds the same was found to be lying between 11.1 and $48.2 \%$. Across different varieties, DPPH free radical scavenging activities from the same component were significantly $(p<0.05)$ different (Table 4). Table 5 indicates the overall contribution of the pulp and seeds to the DPPH free radical scavenging activity of the whole goji. The pulp contributed to $54-94 \%$ of the DPPH for all six varieties, whereas the seeds contribution rate was only $6 \%-46 \%$.

FRAP assay and DPPH free radical scavenging activity assay are two methods to ascertain the antioxidant activity of food and plant. There are colorimetric redox reactions in FRAP assay and DPPH free radical scavenging activity assay. However, in DPPH method, the color is cleared by forming a colorless substance, named DPPH-H. In FRAP method, $\mathrm{Fe}^{3+}$-TPTZ is reduced to $\mathrm{Fe}^{2+}$-TPTZ by the antioxidant compounds which results in blue color.

3.4. Effect of Boiling on the Phytochemical Distribution and Antioxidant Activity of Black and Red Goji. The residual boiled water contained a very relatively high content of phytochemicals and antioxidant activities. This showed that most of the phytochemicals are lost while boiling from the fruits to the water. The boiled fruits had the lowest phytochemicals contents and antioxidant activities. There were some other studies presenting the similar phenomena apart from this work. Attree et al. [12] revealed that boiling leads to significant losses in the phytochemicals and antioxidant activities for peanuts. However, Chukwumah et al. [22] reported the different results that boiling led to an increase in the TPC and TFC as compared to the raw fruits. 
The boiled water had the stronger FRAP values and $\mathrm{DPPH}$ free radical scavenging activity than boiled fruits. It is indicated that most of the antioxidants are lost from the pulp to the water during boiling. This loss may also be attributed to the loss of the phytochemicals from the pulp to the water during boiling. However, Attree et al. [12] investigated the different results in peanuts. It is then speculated that the reverse phenomena were depending on different materials.

\section{Conclusions}

The pulp of the goji had the highest concentration of phytochemicals (TPC, TFC, MAC, and CTC) as compared to the seeds and whole fruits. Not only pulp had the highest concentration of all phytochemicals, but also the contribution rate of pulp was also higher as compared to the seeds except for varieties 3 and 4 for CTC and MAC. Similar observations were obtained for the antioxidant activities with the order being: pulp > seeds > whole fruits. A strong correlation was noted among phytochemicals, antioxidant activities with the goji color. Black goji berries were found to have higher TPC, TFC, MAC, CTC, FRAP, and DPPH than the red goji berries. This work also showed that boiling results in significant $(p$ $<0.05$ ) losses in the phytochemical contents and antioxidant activities. From a nutritional point of view, the consumption of black goji is recommended to maximize the dietary intake of antioxidant compounds. And the health benefits of black and red goji need to be further studied.

\section{Abbreviations}

CAE: (+)-Catechin equivalents

CTC: Condensed tannin content

CyE: Cyanidin-3-glucoside equivalents

DPPH: 2,2-Diphenyl-1-picrylhydrazyl radical

FRAP: Ferric reducing antioxidant power

GAE: Gallic acid equivalents

MAC: Monomeric anthocyanin content

TFC: Total flavonoid content

TPC: Total phenolic content

TPTZ: 2,4,6-Tri(2-pyridyl)-striazine.

\section{Conflicts of Interest}

The authors declare that there are no conflicts of interest regarding the publication of this paper.

\section{Acknowledgments}

This study was jointly supported by two research grants from Beijing Normal University-Hong Kong Baptist University United International College (R201624 and R201714).

\section{References}

[1] O. Potterat, "Goji (Lycium barbarum and L. chinense): phytochemistry, pharmacology and safety in the perspective of traditional uses and recent popularity," Planta Medica, vol. 76, no. 1, pp. 7-19, 2010.
[2] J. Zhao, H. Li, W. Xi et al., "Changes in sugars and organic acids in wolfberry (Lycium barbarum L.) fruit during development and maturation," Food Chemistry, vol. 173, pp. 718-724, 2015.

[3] Y. Liu, S. Zeng, W. Sun et al., "Comparative analysis of carotenoid accumulation in two goji (Lycium barbarum L. and L. ruthenicum Murr.) fruits," BMC Plant Biology, vol. 14, no. 1, article 269, 2014.

[4] N. He, X. Yang, Y. Jiao, L. Tian, and Y. Zhao, "Characterisation of antioxidant and antiproliferative acidic polysaccharides from Chinese wolfberry fruits," Food Chemistry, vol. 133, no. 3, pp. 978-989, 2012.

[5] D. B. Lin, Y. Jiang, L. Wark, H. He, L. Willard, and D. Medeiros, "Dietary wolfbery increases hepatic insulin sensitivity in obese mice," The FASEB Journal, vol. 6, no. 3, pp. 828-837, 2012.

[6] P. H. W. Chu, H.-Y. Li, M.-P. Chin, K.-F. So, and H. H. L. Chan, "Effect of Lycium Barbarum (Wolfberry) Polysaccharides on preserving retinal function after partial optic nerve transection," PLoS ONE, vol. 8, no. 12, Article ID e81339, 2013.

[7] Q. Luo, Y. Cai, J. Yan, M. Sun, and H. Corke, "Hypoglycemic and hypolipidemic effects and antioxidant activity of fruit extracts from Lycium barbarum," Life Sciences, vol. 76, no. 2, pp. 137-149, 2004.

[8] S. Magiera and M. Zaręba, "Chromatographic determination of phenolic acids and flavonoids in Lycium barbarum L. and evaluation of antioxidant activity," Food Analytical Methods, vol. 8, no. 10, pp. 2665-2674, 2015.

[9] X. Yao, Y. Peng, L. J. Xu, L. Li, Q. L. Wu, and P. G. Xiao, "Phytochemical and biological studies of lycium medicinal plants," Chemistry \& Biodiversity, vol. 8, no. 6, pp. 976-1010, 2011.

[10] T. Islam, X. Yu, T. S. Badwal, and B. Xu, "Comparative studies on phenolic profiles, antioxidant capacities and carotenoid contents of red goji berry (Lycium barbarum) and black goji berry (Lycium ruthenicum)," Chemistry Central Journal, vol. 11, no. 1, p. 59, 2017.

[11] D. Granato, D. S. Nunes, and F. J. Barba, "An integrated strategy between food chemistry, biology, nutrition, pharmacology, and statistics in the development of functional foods: a proposal," Trends in Food Science \& Technology, vol. 62, pp. 13-22, 2017.

[12] R. Attree, B. Du, and B. Xu, "Distribution of phenolic compounds in seed coat and cotyledon, and their contribution to antioxidant capacities of red and black seed coat peanuts (Arachis hypogaea L.)," Industrial Crops and Products, vol. 67, pp. 448-456, 2015.

[13] R. Dong, Y. Zheng, and B. Xu, "Phenolic profiles and antioxidant capacities of chinese unifloral honeys from different botanical and geographical sources," Food and Bioprocess Technology, vol. 6, no. 3, pp. 762-770, 2013.

[14] J. Lee, R. W. Durst, and R. E. Wrolstad, "Determination of total monomeric anthocyanin pigment content of fruit juices, beverages, natural colorants, and wines by the $\mathrm{pH}$ differential method: collaborative study," Journal of AOAC International, vol. 88, no. 5, pp. 1269-1278, 2005.

[15] B. J. Xu and S. K. C. Chang, "A comparative study on phenolic profiles and antioxidant activities of legumes as affected by extraction solvents," Journal of Food Science, vol. 72, no. 2, pp. S159-S166, 2007.

[16] F.-M. Zhu, B. Du, and J. Li, "Effect of ultrafine grinding on physicochemical and antioxidant properties of dietary fiber from wine grape pomace," Food Science and Technology International, vol. 20, no. 1, pp. 55-62, 2014. 
[17] E. M. Tanvir, M. S. Hossen, M. F. Hossain et al., "Antioxidant properties of popular turmeric (Curcuma longa) varieties from Bangladesh," Journal of Food Quality, vol. 2017, Article ID 8471785, 8 pages, 2017.

[18] D. Granato, V. M. de Araújo Calado, and B. Jarvis, “Observations on the use of statistical methods in food science and technology," Food Research International, vol. 55, no. 2, pp. 137149, 2014.

[19] D. Granato, J. S. Santos, L. G. Maciel, and D. S. Nunes, "Chemical perspective and criticism on selected analytical methods used to estimate the total content of phenolic compounds in food matrices," TrAC-Trends in Analytical Chemistry, vol. 80, pp. 266-279, 2016.

[20] M. E. Ionica, V. Nour, and I. Trandafir, "Polyphenols content and antioxidant capacity of goji fruit (Lycium chinense) as affected by the extraction solvents," South Western Journal of Horticulture, Biology and Environment, vol. 3, no. 2, pp. 121-129, 2012.

[21] A. C. M. Hacke, D. Granato, L. G. Maciel et al., "Jabuticaba (Myrciaria cauliflora) seeds: chemical characterization and extraction of antioxidant and antimicrobial compounds," Journal of Food Science, vol. 81, no. 9, pp. C2206-C2217, 2016.

[22] Y. Chukwumah, L. Walker, B. Vogler, and M. Verghese, "Changes in the phytochemical composition and profile of raw, boiled, and roasted peanuts," Journal of Agricultural and Food Chemistry, vol. 55, no. 22, pp. 9266-9273, 2007. 

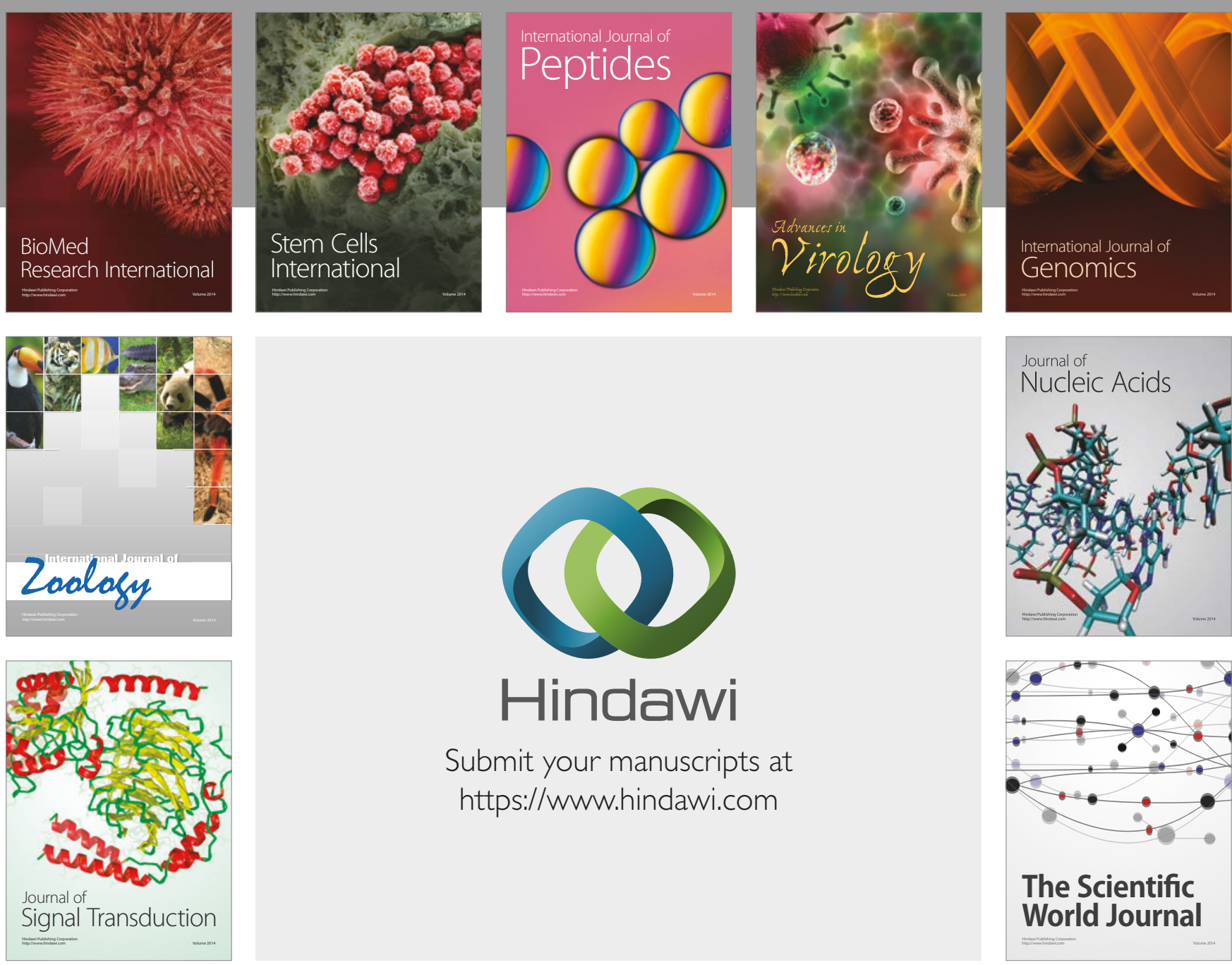

Submit your manuscripts at

https://www.hindawi.com
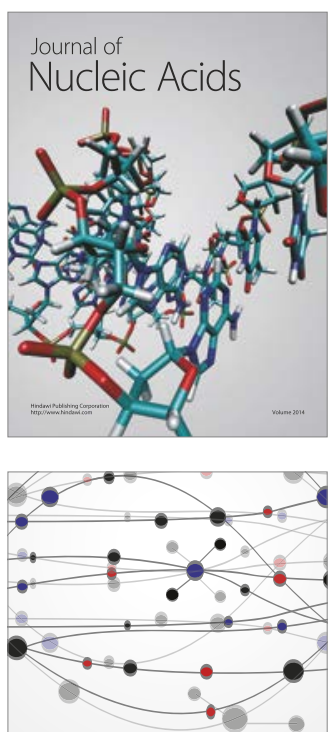

The Scientific World Journal

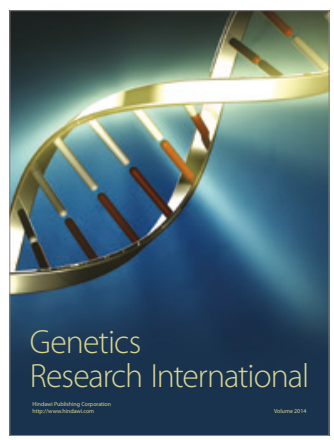

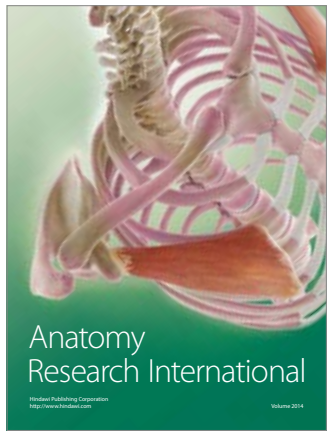

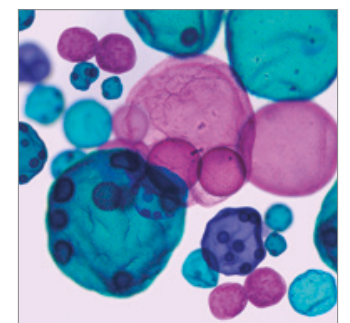

International Journal of Microbiology
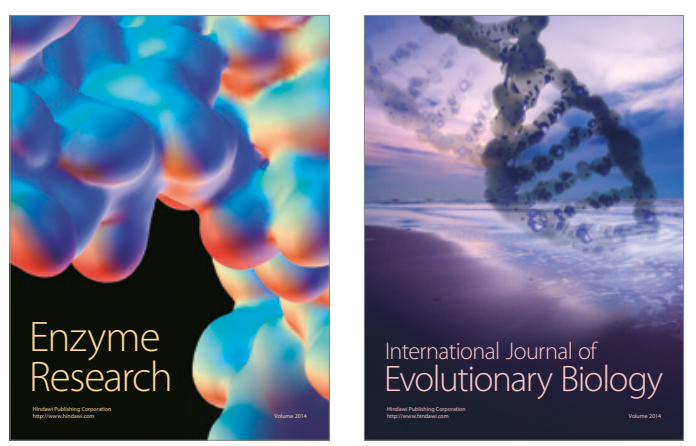
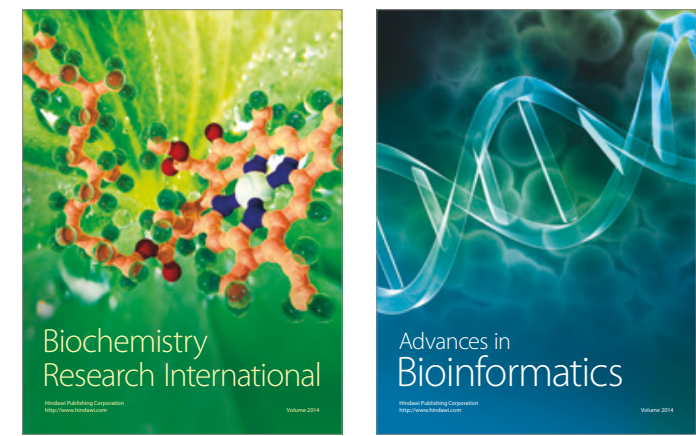

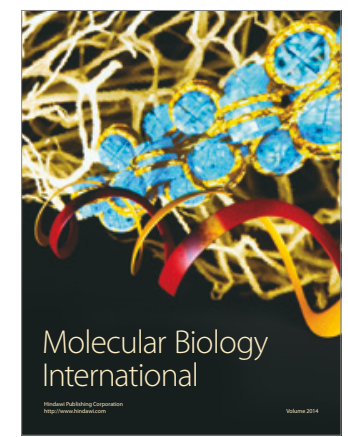

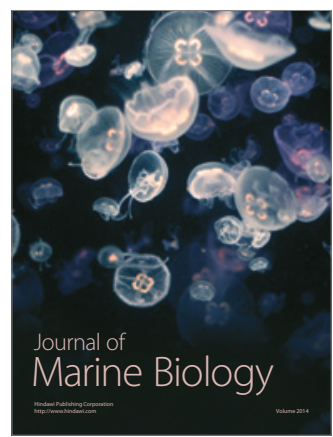

